Preprints of the

Max Planck Institute for

Research on Collective Goods

Bonn 2010/25

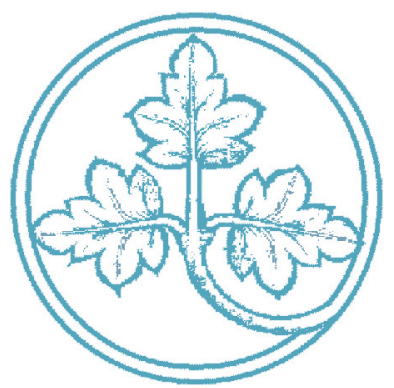

Job Assignment with

Multivariate Skills

Stefanie Brilon

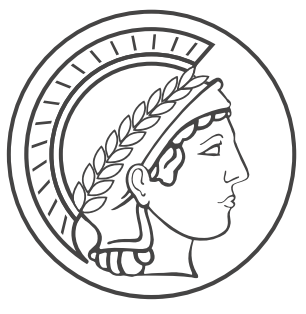




\title{
Job Assignment with Multivariate Skills
}

\author{
Stefanie Brilon
}

May 2010 


\title{
Job Assignment with Multivariate Skills *
}

\author{
Stefanie Brilon
}

May 2010

\begin{abstract}
This paper analyzes the job assignment problem faced by a firm when workers' skills are distributed along several dimensions and jobs require different skills to varying extent. I derive optimal assignment rules with and without slot constraints, and show that under certain circumstances workers may get promoted although in their new job they are expected to be less productive than in their old job. This can be interpreted as a version of the Peter Principle which states that workers get promoted up to their level of incompetence.
\end{abstract}

Keywords: job assignment, worker selection, internal hiring, Peter Principle, slot constraints, multi-dimensional skills.

JEL Classification: J0, J62, M12, M51.

\section{Introduction}

Under which circumstances can performance in one job act as an indicator for performance in another job? Why would an employer want an employee to work first in job 1 before letting him do job 2? Two explanations seem plausible: (i) worker selection: by observing a worker's performance in job 1, the employer learns more about the worker's ability; and (ii) training: Working in job 1 provides the worker with training and skills that are useful in job 2 .

*I thank Martin Hellwig for many helpful discussions, as well as Felix Bierbrauer, Christoph Engel, Guido Friebel, Alia Gizatulina, Baptiste Massenot, Tobias Salz, Konrad Stahl, and Philipp Weinschenk for their comments and suggestions. 
In the following, I concentrate on the first option. To do so, I abstract from any considerations concerning wage costs or workers' incentives to exert effort. Instead, I focus on the employer's task assignment problem when workers vary in their skill levels and different tasks require different combinations of skills. Workers in the model are characterized by a skill combination $(x, z)$ where $x$ and $z$ are two different skills that are independently distributed across the worker population. However, only a worker's overall performance in a given task is observable, not his exact skill profile. The employer therefore faces the problem of how to allocate workers to tasks that put different weights on both skills.

The first part of the paper analyzes the task assignment of current and new workers if the employer faces no constraints concerning the number of workers he can assign to each task. I derive assignment rules and show that there may be a tradeoff between maximizing short-term and long-term output when new workers are hired for two periods: in the short run, output is maximized by assigning new workers to the task where the expected output of an unscreened worker is maximal. However, if this task plays a much more important role in the overall production of the firm, the employer may prefer first to hire workers for the other task, which is thus used as a screening stage for maximizing output in the long run. That is, the employer will prefer to forego some first-period output in order to make more informed choices in the long run.

The second part of the paper abandons the assumption of unconstrained assignment possibilities. At least in the short run, firms often need a fixed number of workers in each task, i.e., there is a given number of jobs in each activity. Given such slot constraints, the second part of the paper determines under what circumstances workers are reallocated between jobs. It thus describes situations in which firms prefer internal over external hiring of workers.

Furthermore, the analysis provides theoretical evidence for a version of the Peter Principle, after Peter and Hull (1969), which states that workers are promoted up to their level of incompetence. In the present model, something similar may arise over a certain range of output realizations: workers producing output in this range will get reallocated although, at least in expected terms, they will be less productive at their new job. Nevertheless, the reallocation of these workers is efficient: when the employer has to fill an open position, he prefers to reallocate a mediocre worker on whom he has at least some information, rather than hiring an unknown worker.

The paper is structured as follows: the following section summarizes the re- 
lated literature. Section 3 describes the optimal task assignment of workers when there are two tasks that require two skills, and there are no slot constraints. This latter assumption is relaxed in Section 4, which analyzes under which circumstances workers are reallocated between jobs if there is a fixed number of workers needed in each job. Additionally, Section 5 generalizes the model to $J$ jobs and $I$ skills, and Section 6 discusses assignment patterns such as job rotation. Section 7 concludes.

\section{Related Literature}

Although there is a large literature on job assignment, as nicely summarized in Sattinger (1993) and Valsecchi (2000), most papers on the topic assume that workers' abilities vary along a single dimension ("general ability") but do not take into account that workers may possess many skills that matter for their performance. Others note that workers may have a comparative advantage in one task, but then often reduce the analysis to a problem with only two types. Only recently, the concept of task-specific skills has been introduced by Gibbons and Waldman (2004, 2006). Building on this concept, Gathmann and Schonberg (2010) analyze empirically to what extent skills are transferable across jobs, the underlying idea being that different occupations combine tasks and thus task-specific skills in different ways.

Like Gathmann and Schonberg (2010), the present paper uses the idea that workers possess many skills and that their skill profile matters for their performance in different tasks. It then derives conditions under which employers prefer to recruit workers internally.

There is a lot of empirical evidence that firms indeed tend to hire internally, as for instance shown in DeVaro and Morita (2009), in Agrawal, Knoeber, and Tsoulouhas (2006), and in Lauterbach, Vu, and Weisberg (1999). The theoretical literature on this topic has proposed several explanations for this phenomenon. For instance, Chan (1996) stresses the incentives created through promotions as a reason for limiting external hiring which would decrease the chances of promotion of current employees. ${ }^{1}$ Another explanation is given by Greenwald (1986), who proposes a model where employers prefer internal hiring because they know the ability of workers they have employed for one

\footnotetext{
${ }^{1}$ Waldman (2003), in a similar setup, points out that the firm may face a time inconsistency problem: ex ante, the incentive aspect is more important, but at the date of promotion, i.e., when effort has already been spent, the assignment aspect becomes more important. That may cause a time inconsistency problem which can be resolved by establishing an internal labor market.
} 
period. Finally, Demougin and Siow (1994) show that, with positive hiring costs, firms may prefer to train and screen workers themselves. By contrast, no such costs are needed in the present model to make internal recruiting attractive: it is enough that the employer has slightly better information about current employees compared to new ones. ${ }^{2}$

Finally, this paper is linked to other models on the Peter Principle such as Fairburn and Malcomson (2001), Faria (2000), and Lazear (2004), which is closest to the present paper. Lazear (2004) explains the Peter Principle as the outcome of a statistical process that displays regression to the mean. By contrast, this paper shows that workers may get reallocated in the first place, although they are expected to be less competent after reallocation. Rather than being a statistical matter, the Peter Principle hence arises as a consequence of incomplete information about job candidates.

\section{Optimal Task Assignment}

Suppose there are two kinds of activities or tasks in a firm. A worker engaged in either of these two activities produces an output $y_{j}, j=1,2$, according to the following production functions

$$
\begin{aligned}
& y_{1}=\alpha \cdot x+(1-\alpha) \cdot z \\
& y_{2}=\beta \cdot x+(1-\beta) \cdot z,
\end{aligned}
$$

where $x$ and $z$ are two different skills needed in both activities, and $\alpha$ and $\beta$ are the weights assigned to skill $x$, with $\alpha, \beta \in[0,1]$.

Skills $x$ and $z$ can, for instance, be thought of as technical and analytical skills respectively. Let us assume that skill $x$ is more important in activity 1 than in activity 2, whereas it is the other way round for skill $z$. That is, the following assumption holds:

\section{Assumption $1 \alpha>\beta$.}

The cross-section distribution of the two skills is assumed to be normal with $x \sim N\left(\mu_{x}, \sigma_{x}\right)$ and $z \sim N\left(\mu_{z}, \sigma_{z}\right)$, and zero correlation between the two skills. These statistical properties are common knowledge. Furthermore, the employer can observe the output produced by a worker, but not the worker's specific skill level $(x, z)$. The employer can hire workers for 2 periods of time 


$\begin{array}{cccc} & & & \\ \text { Initial task } & \text { Observe } & \text { Reassignment? } & \text { Observe } \\ \text { assignment } & \text { first period } & & \text { Tim } \\ \text { to task } j & \text { output } \hat{y}_{j} & & \text { output }\end{array}$

Figure 1: Time line.

at most and may reassign them after the first period. That is, upon hiring a worker he faces a sequence of decisions as shown in Figure 1.

For the rest of this section, it is assumed that the employer may assign any number of workers to any of the two tasks. That is, the employer faces no slot constraints in his allocation problem but can concentrate on the question how a given worker is optimally allocated to one of the two tasks.

The resulting allocation problem is of course different for workers whose performance in one of the two tasks has already been observed and for new workers on whom the employer has no special information. The following two sections therefore consider these two considerations in turn.

\subsection{Internal Reallocation of Current Workers}

Let us first consider the allocation decision of an employer who has observed workers for one period. That is, we look at the (re-)allocation problem the employer faces after the first period in Figure 1.

In order to decide whether a worker should be reallocated or not, the employer will have to calculate the worker's expected performance in either task given his performance so far.

For instance, a worker whose observed output in task 1 has been $\hat{y}_{1}$ can be expected to produce $E\left[y_{2} \mid \hat{y}_{1}\right]$ as follows if reassigned to task 2 :

$$
E\left(y_{2} \mid \hat{y}_{1}\right)=E\left[y_{2}\right]+k_{1} \cdot\left(\hat{y}_{1}-E\left[y_{1}\right]\right)
$$

where $k_{1}$ is defined as

$$
k_{1}:=\frac{\alpha \beta \sigma_{x}^{2}+(1-\alpha)(1-\beta) \sigma_{z}^{2}}{\alpha^{2} \sigma_{x}^{2}+(1-\alpha)^{2} \sigma_{z}^{2}}=\frac{\operatorname{Cov}\left(y_{1}, y_{2}\right)}{\operatorname{Var}\left(y_{1}\right)}
$$

\footnotetext{
${ }^{2}$ Still, the model in Demougin and Siow (1994) is in some respects similar to the analysis presented here, for instance in its consideration of slot constraints.
} 
That is, $k_{1}$ is equal to the ratio between the covariance of output in tasks 1 and 2 and the variance of output in task 1 . For more information on the calculation of the conditional expected output, see the Appendix.

Suppose that the employer wants to maximize the overall sum of outputs $y_{1}$ and $y_{2}$. Then it is efficient to reallocate an employee if

$$
E\left[y_{2} \mid \hat{y}_{1}\right]>\hat{y}_{1}
$$

i.e., if the expected performance of a worker in task 2 is higher than his observed current performance in task $1, \hat{y}_{1}$. Taking into account expression (1), one can rewrite the above inequality as

$$
\left(k_{1}-1\right) \hat{y}_{1} \geq k_{1} E\left[y_{1}\right]-E\left[y_{2}\right] .
$$

As a consequence, there exists a critical value $\tilde{y}_{1}$ of the form

$$
\tilde{y}_{1}=\left[k_{1} E\left[y_{1}\right]-E\left[y_{2}\right]\right] \frac{1}{k_{1}-1},
$$

such that, if $k_{1}>1$, then reallocation is efficient if the observed output $\hat{y}_{1}$ is higher than $\tilde{y}_{1}$. Note that $k_{1}>1$ if

$$
\sigma_{z}^{2}>\frac{\alpha}{1-\alpha} \sigma_{x}^{2}
$$

that is, if the variance of skill $z$ in the population is high enough or $\alpha$ is sufficiently low or both. If this is true, i.e., if $k_{1}>1$, then the best performing workers in task 1 are likely to be even better in task 2 .

Otherwise, for $k_{1}<1$, reallocation is efficient if $\hat{y}_{1}$ is smaller than $\tilde{y}_{1}$. Then only the worst performers in task 1 are likely to do better in task 2 . This is the case if $\sigma_{z}^{2}<\frac{\alpha}{1-\alpha} \sigma_{x}^{2}$.

By a similar reasoning, a reallocation from task 2 to task 1 is efficient if the worker's task 2 output is higher (lower) than a critical output level

$$
\tilde{y}_{2}=\left[k_{2} E\left[y_{2}\right]-E\left[y_{1}\right]\right] \frac{1}{k_{2}-1},
$$

for $k_{2}$ larger (smaller) than 1 , where $k_{2}$ is defined as

$$
k_{2}:=\frac{\operatorname{Cov}\left(y_{1}, y_{2}\right)}{\operatorname{Var}\left(y_{2}\right)}=\frac{\alpha \beta \sigma_{x}^{2}+(1-\alpha)(1-\beta) \sigma_{z}^{2}}{\beta^{2} \sigma_{x}^{2}+(1-\beta)^{2} \sigma_{z}^{2}} .
$$

Note that $k_{2}$ is larger than 1 if

$$
\sigma_{z}^{2}<\frac{\beta}{1-\beta} \sigma_{x}^{2}
$$




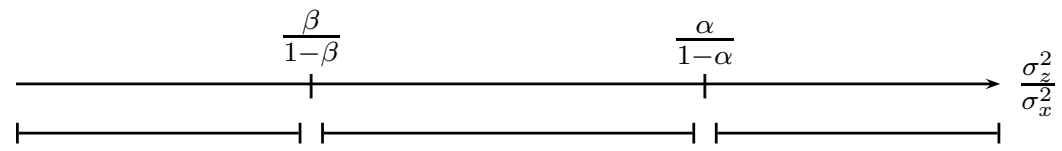

$$
\begin{array}{ccc}
\text { Case } 1 & \text { Case } 2 & \text { Case } 3 \\
k_{1}<1, k_{2}>1 & k_{1}<1, k_{2}<1 & k_{1}>1, k_{2}<1
\end{array}
$$

Figure 2: Possible combinations for $k_{1}$ and $k_{2}$, depending on $\sigma_{z}^{2} / \sigma_{x}^{2}$.

By definition, one has

$$
k_{1} \cdot k_{2}=\frac{\left(\operatorname{Cov}\left(y_{1}, y_{2}\right)\right)^{2}}{\operatorname{Var}\left(y_{1}\right) \cdot \operatorname{Var}\left(y_{2}\right)}=\varrho_{y_{1} y_{2}}^{2} .
$$

Since the correlation coefficient $\varrho_{y_{1} y_{2}}$ can be at most equal to one, it follows directly that the variables $k_{1}$ and $k_{2}$ cannot both at the same time be larger than 1.

Therefore the following proposition holds:

Proposition 1 For $k_{1}>1$, there exists a critical task 1 output level $\tilde{y}_{1}$ as defined in (3) such that for $y_{1}>\tilde{y}_{1}$ it is efficient to reallocate a worker from task 1 to task 2. At the same time, there exists a critical task 2 output level $\tilde{y}_{2}$ as defined in (5) such that for $y_{2}<\tilde{y}_{2}$ it is efficient to reallocate workers from task 2 to task 1.

As a corollary to this proposition, we get that if $k_{2}>1$, then it is efficient to reallocate workers with an observed output $\hat{y}_{1}<\tilde{y}_{1}$ to task 2 and workers with $\hat{y}_{2}>\tilde{y}_{2}$ to task 1 .

Furthermore the case may arise that $k_{1}<1$ and $k_{2}<1$, and hence only the worst performers get reallocated, i.e., workers with $\hat{y}_{1}<\tilde{y}_{1}$ and with $\hat{y}_{2}<\tilde{y}_{2}$. This result also holds when the covariance between tasks 1 and 2 is negative.

Which of these constellations is relevant depends on the relative size of the variances of skill $x$ and $z$. As we have seen, $k_{1}>1$ is equivalent to $\sigma_{z}^{2} / \sigma_{x}^{2}>$ $\alpha /(1-\alpha)$ and $k_{2}>1$ if $\sigma_{z}^{2} / \sigma_{x}^{2}<\beta /(1-\beta)$. Furthermore, given Assumption 1 , we know that $\alpha /(1-\alpha)>\beta /(1-\beta)$. We can thus derive three cases, which are defined as follows:

- Case 1: If $\sigma_{z}^{2} / \sigma_{x}^{2}<\beta /(1-\beta)$, then $k_{1}<1$ and $k_{2}>1$.

- Case 2: If $\beta /(1-\beta) \leq \sigma_{z}^{2} / \sigma_{x}^{2} \leq \alpha /(1-\alpha)$, then $k_{1}<1$ and $k_{2}<1$. 
- Case 3: If $\alpha /(1-\alpha)<\sigma_{z}^{2} / \sigma_{x}^{2}$, then $k_{1}>1$ and $k_{2}<1$.

These cases are also summarized in Figure 2. In Cases 1 and 2, reallocation from task 1 to task 2 takes place if workers produce an output $\hat{y}_{1}<\tilde{y}_{1}$, whereas in Case 3 workers get reallocated to task 2 if they produce $\hat{y}_{1}>\tilde{y}_{1}$. Reallocation from task 2 to task 1 is optimal in Cases 2 and 3 if $\hat{y}_{2}<\tilde{y}_{2}$, and in Case 1 if $\hat{y}_{2}>\tilde{y}_{2}$.

\subsection{Assignment of New Workers}

So far, the reallocation of current workers has been considered. But how about new workers? To which task should they be allocated first? There are two criteria that may possibly play a role: (i) Which task provides the employer with more precise information about the employee? (ii) Where can an unscreened worker be expected to produce more?

If new workers get hired for only one period, then learning about the employee obviously does not play a role, and the employer will therefore prefer to allocate new workers to the task where the expected output of an unknown worker is higher, and thus the second motive dominates. The same is true if workers get hired for several periods, but cannot be reallocated or fired.

If workers instead get hired for two periods and reallocation is possible, then there may be a tradeoff between the two motives, i.e., between learning and output maximization of unscreened workers. To see this, let us make the following assumption: ${ }^{3}$

\section{Assumption $2 E\left[y_{1}\right]>E\left[y_{2}\right]$.}

That is, an unscreened worker is expected to be more productive in task 1. The employer can hire the worker for either of the two tasks $j=1,2$. After one period of work, he will observe the worker's first period output $\hat{y}_{j}$. According to the rules derived in the previous section, he will then either let the worker continue to work on his current task or he will reassign him. Assuming that $k_{1}>1$ and $k_{2}<1$, this means that workers who were first assigned to task 1 get reallocated to task 2 if their conditional expected output in task $2, E\left[y_{2} \mid \hat{y}_{1}\right]$, is greater than their current output, $\hat{y}_{1}$. As shown in Proposition 1 , this is the case if $\hat{y}_{1}$ greater than $\tilde{y}_{1}$ as defined in (3). Similarly, workers who were first hired for task 2 get reassigned to

\footnotetext{
${ }^{3}$ This assumption is used to illustrate clearly the results in the remainder of the paper. All findings still apply if $E\left[y_{1}\right] \neq E\left[y_{2}\right]$, which should hold generically.
} 


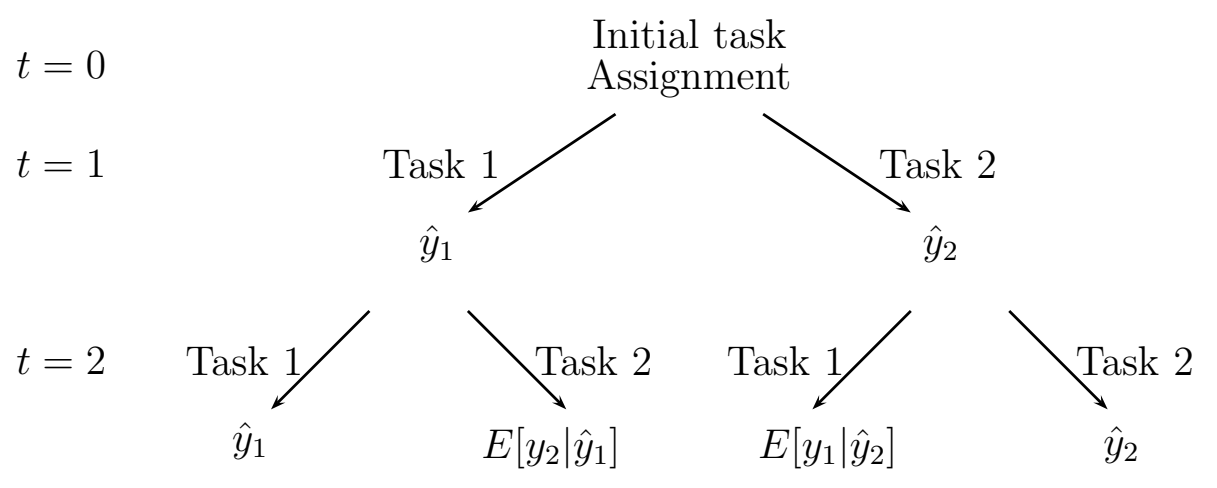

Figure 3: Decision Tree.

task 1 if they are likely to produce a higher output in this position, i.e., if $E\left[y_{1} \mid \hat{y}_{2}\right]>\hat{y}_{2}$. This is the case if $\hat{y}_{2} \leq \tilde{y}_{2}$ which was defined in (5). All other workers continue to work on the same task. Figure 3 illustrates these considerations.

From an ex ante point of view, the expected output of a worker hired for two periods and first assigned to task 1 therefore is

$$
E\left[y_{1}\right]+\left[E\left[y_{1} \mid \hat{y}_{1}<\tilde{y}_{1}\right] \cdot \operatorname{Prob}\left(\hat{y}_{1} \leq \tilde{y}_{1}\right)\right]+\left[E\left[y_{2} \mid \hat{y}_{1}>\tilde{y}_{1}\right] \cdot \operatorname{Prob}\left(\hat{y}_{1}>\tilde{y}_{1}\right)\right],
$$

i.e., the worker's expected first period production in task 1 plus his second period output conditional on his observed performance $\hat{y}_{1}$ in the first period. Note that a worker's performance at a given task does not change over time. That is, his second period output in task 1 is the same as in the first period, $\hat{y}_{1}$. His expected second period performance in task 2 is given by equation (1). Taking this into account, one can rewrite the above expression as

$$
E\left[y_{1}\right]+\left[y_{1} \cdot \operatorname{Prob}\left(y_{1} \leq \tilde{y}_{1}\right)\right]+\left[E\left[y_{2}\right]+k_{1}\left(y_{1}-E\left[y_{1}\right]\right)\right] \cdot \operatorname{Prob}\left(y_{1}>\tilde{y}_{1}\right) .
$$

Let $F(\cdot)$ be the cumulative distribution function of $y_{1} \cdot{ }^{4}$ Then the expected output of a worker hired for two periods is

$$
E\left[y_{1}\right]+\left[F\left(\tilde{y}_{1}\right)\right]+\left[E\left[y_{2}\right]+k_{1}\left(1-F\left(\tilde{y}_{1}\right)\right)-k_{1} E\left[y_{1}\right]\right] .
$$

A similar reasoning applies when workers first work on task 2. After observing their first-period performance $\hat{y}_{2}$, the employer will reassign them to task 1

\footnotetext{
${ }^{4}$ Given the assumptions about $x$ and $z, y_{1}$ is normally distributed with mean $\alpha \mu_{x}+$ $(1-\alpha) \mu_{z}$ and variance $\alpha^{2} \sigma_{x}^{2}+(1-\alpha)^{2} \sigma_{z}^{2}$.
} 
if $\hat{y}_{2} \leq \tilde{y}_{2}$ and otherwise let them continue to work on task 2. From an ex ante point of view, the expected output of a worker hired for two periods and first assigned to task 2 hence is

$$
\begin{aligned}
& E\left[y_{2}\right]+\left[E\left[y_{1} \mid \hat{y}_{2}<\tilde{y}_{2}\right] \cdot \operatorname{Prob}\left(\hat{y}_{2} \leq \tilde{y}_{2}\right)\right]+\left[E\left[y_{2} \mid \hat{y}_{2}>\tilde{y}_{2}\right] \cdot \operatorname{Prob}\left(\hat{y}_{2}>\tilde{y}_{2}\right)\right] \\
= & E\left[y_{2}\right]+\left[E\left[y_{1}\right]+k_{2}\left(y_{2}-E\left[y_{2}\right]\right)\right] \cdot \operatorname{Prob}\left(\hat{y}_{2} \leq \tilde{y}_{2}\right)+\left[y_{2} \cdot \operatorname{Prob}\left(\hat{y}_{2}>\tilde{y}_{2}\right)\right] .
\end{aligned}
$$

Let $G(\cdot)$ be the cumulative distribution function of $y_{2} \cdot{ }^{5}$ Then the above expression can be rewritten as

$$
E\left[y_{2}\right]+\left[E\left[y_{1}\right]+k_{2} G\left(\tilde{y}_{2}\right)-k_{2} E\left[y_{2}\right]\right]+\left[1-G\left(\tilde{y}_{2}\right)\right]
$$

By comparing (7) and (8), we can determine which strategy is more promising, and thus derive the following proposition:

Proposition 2 Given Assumptions 1 and 2, if $k_{1}>1$, then an employer who hires workers for two periods of time will prefer to hire them first for task 1 if

$$
E\left[y_{1}\right]<\frac{k_{2}}{k_{1}} E\left[y_{2}\right]+\frac{k_{1}-1}{k_{1}}\left(1-F\left(\tilde{y}_{1}\right)\right)+\frac{1-k_{2}}{k_{1}} G\left(\tilde{y}_{2}\right),
$$

and for task 2 otherwise.

That is, the employer hires workers first to task 1 only if the expected output in task 1 is not too high compared to task 2. Otherwise, if the unconditional expected output in task 1 is much higher than in task 2 , such that condition (9) no longer holds, the employer puts unscreened workers first on task 2. He does this even though an unscreened worker would be more productive in task 1, at least in expected terms. The employer thus prefers to sacrifice a certain amount of first-period production in order to have more information on workers that are assigned to the task that is likely to generate a higher output. This tradeoff is similar to the one described in Grossman, Kihlstrom, and Mirman (1977), who show that individuals or firms may find it profitable to modify their behavior in order to base their future decisions on better information. That is, they experiment in order to gain information.

The same is true in the present model. It would seem natural that the employer should assign workers to the job where they can be expected to produce the highest output. However, if task 1 is sufficiently more important than task 2 , or if performance in task 2 is much more informative about

\footnotetext{
${ }^{5} y_{2}$ is normally distributed with mean $\beta \mu_{x}+(1-\beta) \mu_{z}$ and variance $\beta^{2} \sigma_{x}^{2}+(1-\beta)^{2} \sigma_{z}^{2}$.
} 
workers' skills, then the employer may find it worthwhile to assign workers first to task 2, thus basically buying information on workers at the cost of a lower expected output in the short run. Or, in the terms of the model by Grossman, Kihlstrom, and Mirman (1977): the employer decides to "experiment" if the benefits from more informed future choices outweigh the costs incurred because he modifies his behavior relative to what would be optimal if there was no learning. ${ }^{6}$

Note, however, that, while it is possible that such "experimentation" arises, it is not necessarily the case. Depending on the exact distributions of $y_{1}$ and $y_{2}$, it may very well be the case that $E\left[y_{1}\right]>E\left[y_{2}\right]$ and at the same time condition (9) is not fulfilled. That is, there is not necessarily a tradeoff between maximizing the first-period output of an unknown worker and learning about his skills. In that case, the employer will be better off assigning new workers to task 1 where they are expected to produce a higher output.

\section{Job Assignment with Slot Constraints}

The previous section has established criteria for assigning both new and old workers to one of two activities in the firm. These criteria are relevant if the employer faces no constraints concerning the number of employees working on each task. However, the employer may not be able to assign any number of workers to any task. At least in the short run he is likely to face slot constraints in the sense that he needs a fixed number of employees in each activity.

Let us assume that the firm needs $n$ workers to do task 1 and $m$ workers for task 2 . That is, there are $n$ jobs of type 1 and $m$ jobs of type 2 . All prices are normalized to one and the firm's objective function is given by

$$
\sum_{i=1}^{n} y_{1}^{i}+\sum_{i=1}^{m} y_{2}^{i},
$$

i.e., the principal wants to maximize the sum of outputs while keeping the number of workers in each task fixed. In the following, only the employer's short-term perspective is considered, i.e., he wants to maximize the sum of outputs in the next period. To fill all available slots, the employer can either reallocate current workers or hire new unscreened workers for either of the two jobs. So when does the firm choose which option?

\footnotetext{
${ }^{6}$ Similar behavior may be found on the side of employees: as Antonovics and Golan (2007) show, if workers don't know their type, they may also engage in experimentation in order to learn more about their skills.
} 


\subsection{External Recruiting vs. Internal Reallocation}

Suppose the firm has to fill one open position in job $2 .^{7}$ Should it fill the position in job 2 with someone who has worked in job 1 before, or should it hire an unknown worker?

If the firm promotes worker $i$ from job 1 to job 2 and fills the opening in job 1 with an unknown worker, its expected profit in the next period is

$$
E\left[y_{1}\right]+E\left[y_{2}^{i} \mid \hat{y}_{1}^{i}\right]
$$

i.e., the sum of the expected output of an unknown worker in job 1 and the expected output of worker $i$ in job 2 , conditional on his observed performance $\hat{y}_{1}^{i}$ in his current job.

If the firm does not promote worker $i$ and hires an unknown worker for job 2 , its expected profits are

$$
\hat{y}_{1}^{i}+E\left[y_{2}\right]
$$

i.e., worker $i$ will continue to produce the same output $\hat{y}_{1}^{i}$ as before, and the new worker has expected job 2 output $E\left[y_{2}\right]$.

Promoting worker $i$ is hence the better option if

$$
\begin{aligned}
E\left[y_{1}\right]+E\left[y_{2}^{i} \mid \hat{y}_{1}^{i}\right] & >\hat{y}_{1}^{i}+E\left[y_{2}\right] \\
\Leftrightarrow E\left[y_{1}\right]+E\left[y_{2}\right]+k_{1}\left(\hat{y}_{1}^{i}-E\left[y_{1}\right]\right) & >\hat{y}_{1}^{i}+E\left[y_{2}\right] \\
\Leftrightarrow\left(k_{1}-1\right) \hat{y}_{1}^{i} & >\left(k_{1}-1\right) E\left[y_{1}\right] .
\end{aligned}
$$

As before, one has to distinguish two cases: if $k_{1}>1$, then promotion is profitable for $\hat{y}_{1}^{i}>E\left[y_{1}\right]$. In the opposite case, i.e., if $k_{1}<1$, promotion is only profitable if the current output is below the expected output in this job, i.e., if $\hat{y}_{1}^{i}<E\left[y_{1}\right]$. The analogue reasoning applies for job 2.

Proposition 3 For $k_{1}>1$, rather than hiring a new worker, the employer prefers to reallocate a current worker from job 1 to job 2 if his output $\hat{y}_{1}>$ $E\left[y_{1}\right]$, and from job 2 to job 1 if $\hat{y}_{2}<E\left[y_{2}\right]$.

As a corollary, we get that for $k_{2}>1$ reallocation from job 1 to 2 is profitable if $\hat{y}_{1}<E\left[y_{1}\right]$ and from job 2 to 1 if $\hat{y}_{2}>E\left[y_{2}\right]$. If both $k_{1}$ and $k_{2}$ are smaller than one, then reallocation is profitable for $\hat{y}_{1}<E\left[y_{1}\right]$ and $\hat{y}_{2}<E\left[y_{2}\right]$.

\footnotetext{
${ }^{7}$ If there is an opening in job 1 , the employer's considerations run along the same lines as those outlined in the following.
} 


\subsection{Internal Recruiting and the Structure of the Firm}

The results so far suggest that internal hiring becomes more likely if the number of positions $n$ and $m$ to fill in task 1 and 2 is very uneven. Suppose, for instance, that $k_{1}>1$ holds $^{8}$ and hence above average workers in task 1 (i.e., with output $\hat{y}_{1}>E\left[y_{1}\right]$ ) are candidates for reallocation to task 2 . The chances that one of the current job 1 workers is above average increases of course with the number of workers $n$ in this job. As a consequence, it becomes more likely that all job 2 workers are recruited internally, if $n$ is relatively high and $m$ relatively low.

Supposing that job 2 ranks above job 1 in a hierarchy, this finding is in line with the empirical literature on internal recruiting, which generally finds that larger firms are more likely to recruit internally. DeVaro and Morita (2009) also show that this is particularly true in more "bottom heavy" firms, i.e., with a lot more workers on lower levels of the hierarchy. A similar finding is also reported by Hutchens (2006), who examines why firms sometimes employ older workers, but tend to not hire new older workers for the same job. He shows that older workers often hold jobs that are simply not filled from the outside, and that this phenomenon is more likely to occur in firms with a larger number of workers in lower-level jobs.

\subsection{The Peter Principle: Inefficient Reallocation?}

The paper has derived two rules for the reassignment of workers: while Section 3.1 has focused on the optimal reassignment of workers without slot constraints, Section 4.1 considered reassignment when there is a limited number of positions in each task that have to be filled.

When we compare the two resulting reassignment rules, it becomes clear that they usually do not coincide: if there are no constraints on the number of workers in each task, the optimal assignment of a worker currently employed in task $j=1,2$ depends on his performance relative to a critical output level $\tilde{y}_{j}$ as summarized in Proposition 1. By contrast, if there are slot constraints, what matters is just the worker's performance relative to the average performance of an unscreened worker, i.e., $E\left(y_{j}\right)$, as shown in Proposition 3.

Take, for example, the case where $k_{1}>1$ and Assumption 2 holds, i.e., $E\left[y_{1}\right]>E\left[y_{2}\right] .{ }^{9}$ In this case, which is illustrated in Figure 4, $\tilde{y}_{1}>E\left[y_{1}\right]$.

\footnotetext{
${ }^{8}$ This corresponds to Case 3 as defined in Section 3.1.

${ }^{9}$ This corresponds to Case 3 in the above.
} 


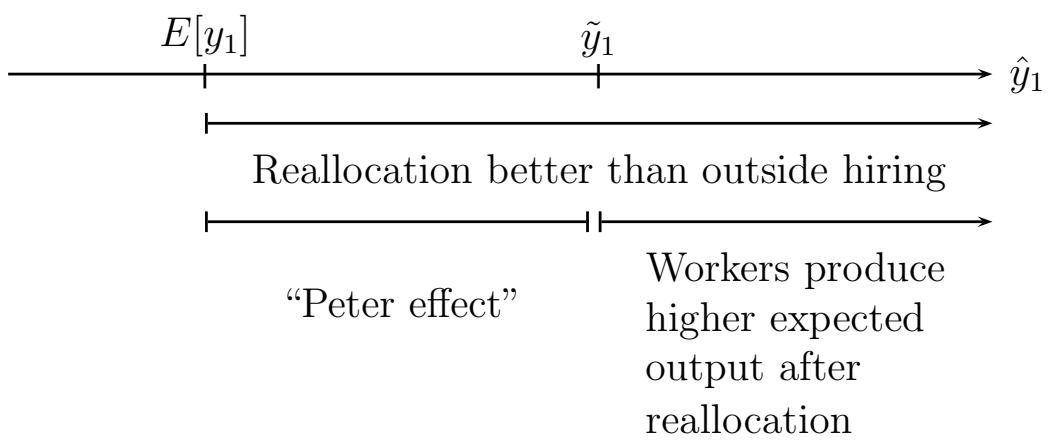

Figure 4: Individual efficiency and profitability of reallocation for $k_{1}>1$.

Any worker producing an output above $E\left[y_{1}\right]$ is a candidate for reallocation according to Proposition 3. However, only workers whose output also exceeds $\tilde{y}_{1}$ can be expected to be more productive after reallocation according to Proposition 1. That is, workers producing output $\tilde{y}_{1}>\hat{y}_{1}>E\left[y_{1}\right]$ are candidates for reallocation according to Proposition 3, even though they are likely to be less productive after reallocation than they are in their current job.

This immediately brings to mind the well-known Peter Principle ${ }^{10}$ which states that workers get promoted up to their level of incompetence. If this principle applies in the present model, then after reallocation, there must be some workers who are less competent or less productive in their new job than they were in their previous job.

This "Peter effect" can indeed be found for workers who get reallocated from job 1 to job 2 if Assumption 2 holds, no matter what the size of of $k_{1}$ and $k_{2} \cdot{ }^{11}$ Workers producing an output level between $\tilde{y}_{1}$ and $E\left[y_{1}\right]$ will be candidates for reallocation to job 2, even though they are likely to perform worse after reallocation. In other words: given their observed performance in job 1, these workers are likely to be more productive or "more competent" in their current job. Nevertheless, they may get transferred to job 2. The model therefore predicts indeed that there are some workers who get reallocated although they can be expected to be less productive or less competent afterwards, as suggested by the Peter Principle.

\footnotetext{
${ }^{10}$ After Peter and Hull (1969).

${ }^{11}$ Note that if Assumption 2 does not hold, then the Peter effect appears for workers who are candidates for reallocation from job 2 to job 1 . The effect only depends on the relative size of $E\left[y_{1}\right]$ and $E\left[y_{2}\right]$.
} 
Proposition 4 For $j=1,2$ and $E\left[y_{j}\right]>E\left[y_{-j}\right]$, workers in job $j$ who produce an output between $\tilde{y}_{j}$ and $E\left[y_{j}\right]$ are candidates for reallocation to $j o b-j$ even though they are expected to be less productive after reallocation.

This effect becomes more relevant the further apart the two critical values $E\left[y_{j}\right]$ and $\tilde{y}_{j}$ are that determine reallocation from job $j$ to job $-j$. The distance between these two values is bigger the lower the coefficient $k_{j}$, and the larger the difference in expected output between the two jobs, i.e., the larger $\left|E\left[y_{1}\right]-E\left[y_{2}\right]\right|$.

In the previous literature on the Peter Principle, Fairburn and Malcomson (2001) discuss how promotions can limit the effect of influence activities of workers and show that depending on the degree of risk aversion, promotions may take place that are not justified by reasons of job assignment (what the authors refer to as the "Peter Principle effect"). In Lazear (2004), the Peter Principle is explained as follows: workers' performance depends both on ability and other random factors such as luck which exhibit regression to the mean. As a consequence, workers' expected performance after promotion is lower than their observed performance on which the promotion decision is based. Furthermore, after promoting the best job 1 workers, the average performance of job 1 workers left behind is lower than it was before. That is, ability appears lower after promotion purely as a statistical matter.

The present model proposes a different explanation. It shows that workers get promoted as soon as they produce more than the average unscreened worker, even if they are likely to be less able and hence less productive after promotion. This is due to the fact that the employer faces an opportunity cost of hiring an unknown worker, and therefore always prefers to fill an open position with a worker on whom he has at least some (positive) information.

Note however, that this is an efficient assignment policy. The Peter Principle here occurs as a by-product of a labor market where workers' skills are difficult to assess. If the employer's information on outside candidates ${ }^{12}$ improves, the scope for the Peter effect as described above diminishes.

\section{Generalization of the Model}

In the basic model described above the weights of skills in a given task always summed up to one, thus putting the focus on the relative importance of skills

\footnotetext{
${ }^{12}$ For instance, the employer may be able to observe past performance of workers with a different employer or he may get a signal on a worker's skill level in one of the relevant skills.
} 
in each task. However, all the results still hold if this is not true. Also, the model can be further generalized to encompass both more tasks and skills, as well as external shocks: suppose that there are $j=1, . ., J$ possible tasks and $i=1, \ldots, I$ skills. Each skill has a different weight $c_{i j}$ in each task. Furthermore let $y_{j}$ denote the output in task $j$. The production function for task $j$ then can be written as

$$
y_{j}=\sum_{i=1}^{I} c_{i j} \cdot x_{i}+\epsilon_{j}
$$

where $x_{i}$ denotes the endowment of a worker with skill $i$, and $\epsilon$ is a noise term. Skills are assumed to be independently distributed in the population according to $N\left(\mu_{x_{i}}, \sigma_{x_{i}}\right)$. The noise term is also assumed to be independently distributed across tasks and across time periods according to $N\left(0, \sigma_{\epsilon}\right)$. This generalization allows for external productivity shocks, which makes it better comparable to existing models of job assignment.

The analysis from the previous section also applies to this more general version of the model: given an observed output in task $j, \hat{y}_{j}$, the expected output of a worker in task $k \neq j$ is

$$
E\left[y_{k} \mid \hat{y}_{j}\right]=E\left[y_{k}\right]+\frac{\operatorname{Cov}\left(y_{j}, y_{k}\right)}{\operatorname{Var}\left(y_{j}\right)} \cdot\left[\hat{y}_{j}-E\left[y_{j}\right]\right] .
$$

If kept in the same task for another period, the worker has an expected output of

$$
E\left[y_{j} \mid \hat{y}_{j}\right]=E\left[y_{j}\right]+\frac{\operatorname{Cov}\left(y_{j}, y_{j}\right)}{\operatorname{Var}\left(y_{j}\right)} \cdot\left[\hat{y}_{j}-E\left[y_{j}\right]\right],
$$

where - in a slight abuse of notation $-\operatorname{Cov}\left(y_{j}, y_{j}\right)$ denotes the covariance of output in the same task across periods.

If there are no slot constraints, then a worker should be reallocated to task $k$ whenever his expected output in task $k$ as given in (10) is greater than his expected output in task $j$, as given in (11). That is, as before, one can determine an output level $\tilde{y}_{(j, k)}$ that is critical for the decision whether a worker should be kept in task $j$ or whether he can be expected to be more productive in task $k$.

If, however, there are slot constraints and the employer has to decide between hiring new workers and worker reallocation, then this decision is determined by workers' performance in task $j, \hat{y}_{j}$, relative to the expected performance of a new worker, $E\left[y_{j}\right]$. 
That is, as in the basic model, we get two critical values that drive the reallocation decision, depending on whether there are slot constraints or not, namely $\tilde{y}_{(j, k)}$ and $E\left[y_{j}\right]$. These two values will usually not coincide, and we thus can get the same effects as in the basic model. In particular, the Peter effect described above may still occur.

\section{Career Paths}

In the model presented here, the employer can only learn more about a worker's type if he assigns the worker to different jobs. This raises the questions how an employer wants to structure a possible career path. Will consecutive jobs be closely related to each other? When is job rotation a useful mechanism?

Consider the following specification of the model: there are four jobs that use four different skills as follows:

$$
\begin{aligned}
& y_{1}=c_{11} x_{1}+c_{12} x_{2} \\
& c_{22} x_{2}+c_{23} x_{3} \\
& y_{2}=
\end{aligned}
$$

Suppose that job 4 is a management job. In order to choose candidates for this job, the employer faces the option of letting a worker move gradually through jobs 1, 2, and 3, i.e., structure his career path as a job ladder. Or he may want to let workers do job 1 and 3 before moving them to job 4, i.e., implement a job rotation between quite different jobs (1 and 3).

The latter structure allows the employer to extract the most information about the worker in just two time periods. However, switching a job 1 worker to job 3 promises the same expected job 3 output as hiring a completely new worker for the job. Also, by letting a worker work his way through job 1 to 3 , the employer gets more observations and hence a better estimate of the worker's skills.

A job ladder hence provides the employer with a more thorough candidate screening, but it also takes more time. A job rotation program, by contrast, is a speedier way to collect some information about aspiring managers, though at the cost of possibly getting a very low performance of workers in job 3 .

This cost can, however, be mitigated if the employer has better information about workers before hiring them for a job rotation program. Supposing 
workers can signal their skills through education, previous work, extracurricular activities, and so on, workers with good signals from various fields therefore seem to be more likely to be selected into job rotation schemes, such as, for example, high-profile trainee programs. ${ }^{13}$

Of course, the analysis in this paper also neglects a second aspect that may play an important role here, namely that the structuring of a career path also affects the learning process of the employee. This aspect is analyzed in Gibbons and Waldman (2004), who introduce task-specific human capital that is acquired through learning by doing. ${ }^{14}$ Reallocating workers to similar jobs, i.e., specialization, hence makes the most efficient use of this capital. However, for a manager it may be more important to be somewhat knowledgeable in several fields, rather than being an expert in just one, which would explain the existence of job rotation.

So which of these two explanations - learning by the employer or learning by the employee - goes the longer way in explaining why firms adopt job rotation practices? The papers by Ortega (2001) and Eriksson and Ortega (2006) consider exactly this question. ${ }^{15}$ While they find evidence that both employer and employee learning may play a role, the evidence for the former seems slightly stronger. In particular, the authors find that tenure in the firm has a significant negative effect on rotation, whereas tenure in the industry does not, thus suggesting that rotation is a means to get to know the employee, rather than a training device. Furthermore, firms with more hierarchy levels and broader recruiting strategies, as well as growing firms, are more likely to adopt job rotation, all of which supports the employer learning hypothesis.

\section{Conclusion}

The paper proposed a simple task assignment model with multidimensional skills and derived conditions, under which the employer can increase output by reallocating workers. However, there is a potential tradeoff between short-term output maximization and learning about the employee's skills. If

\footnotetext{
${ }^{13}$ This would correspond to the results of the studies by Campion, Cheraskin, and Stevens (1994) and Kusunoki and Numagami (1998), which both suggest that rotation may be good for a worker's career and possibly is used to generate the promotion pool for new managers.

${ }^{14}$ Note that this is an important difference to the present model, where workers' skill levels are given from the outset and at best may be improved through learning by doing.

${ }^{15}$ Ortega (2001) and Eriksson and Ortega (2006) also identify a third motive for job rotation, namely motivating employees by mitigating boredom. However, they find little evidence in support of this motive.
} 
one task is sufficiently important, then the employer may be interested in choosing workers for this task in a more sophisticated way. For instance, he can get more information on these workers by first assigning them to another task with lower expected output, thus reducing his short-run expected output for the sake of making a more informed choice in the future.

While this first part of the paper thus looked at the optimal assignment of a given worker if the employer is free to assign him to any task he sees fit, the second part considered a setting where the employer has to fill a given job with a worker which he may choose from within or outside the firm. In such a setting, workers may get reassigned to another job even if, in expectation, they will be less productive after reassignment. This simply arises because employers prefer to reallocate workers on whom they have some information, rather than hire completely new workers on the market.

The paper thus provides a new explanation for the Peter Principle: workers may indeed get reallocated in such a way that, in the end, they are actually less suited for their current than for their previous job. However, this policy is efficient, since the employer otherwise has to fill his open positions with new applicants on whom he has less information than on his current workers. The Peter Principle thus arises as a by-product of insufficient knowledge about outside candidates and its relevance varies with the availability of information on workers.

Whether this effect plays a role therefore depends crucially on the transparency of the relevant labor market. The transparency of the labor market, in turn, and hence the information available on candidates, depends on market structures, the degree of competition, and the availability of reliable signals on workers' capabilities. While these aspects are beyond the current model, it may be interesting to explore them further. 


\section{Appendix}

\section{Conditional Normal Distributions: General Theorem}

How to calculate the conditional expectation of $x$ and $z$ ? A nice summary is provided in Greene (2003): Let $\mathbf{x}=\left(x_{1}, x_{2}, \cdots, x_{n}\right)^{\prime}$ be a vector of $n$ random variables with mean vector $\boldsymbol{\mu}$ and covariance matrix $\boldsymbol{\Sigma}$. Let $\mathbf{x}_{\mathbf{1}}$ be any subset of variables, and let $\mathbf{x}_{\mathbf{2}}$ be the remaining variables. Likewise, partition $\boldsymbol{\mu}$ and $\Sigma$ so that

$$
\boldsymbol{\mu}=\left(\begin{array}{l}
\mu_{1} \\
\mu_{2}
\end{array}\right)
$$

and

$$
\Sigma=\left(\begin{array}{ll}
\Sigma_{11} & \Sigma_{12} \\
\Sigma_{21} & \Sigma_{22}
\end{array}\right)
$$

Then the conditional distribution of $\mathbf{x}_{\mathbf{1}}$ given $\mathbf{x}_{\mathbf{2}}$ is normal as well:

$$
\boldsymbol{x}_{\mathbf{1}} \mid \boldsymbol{x}_{\mathbf{2}} \sim N\left(\mu_{1.2}, \Sigma_{11.2}\right)
$$

with

$$
\begin{aligned}
\mu_{1.2} & =\mu_{1}+\Sigma_{12} \Sigma_{22}^{-1}\left(x_{2}-\mu_{2}\right) \\
\Sigma_{11.2} & =\Sigma_{11}-\Sigma_{12} \Sigma_{22}^{-1} \Sigma_{21}
\end{aligned}
$$

\section{Application to the Production Function}

Consider the production function $y=a x+b z$, where variables $x$ and $z$ are normally distributed. ${ }^{16}$ The production function can be rewritten in matrix notation as

$$
\left(\begin{array}{l}
x \\
z \\
y
\end{array}\right)=\underbrace{\left(\begin{array}{ll}
1 & 0 \\
0 & 1 \\
a & b
\end{array}\right)}_{:=A}\left(\begin{array}{l}
x \\
z
\end{array}\right)
$$

The covariance matrix $\hat{\Sigma}$ of this vector of normally distributed variables is calculated as $A \Sigma A^{\prime}{ }^{17}$ where $A$ is the matrix of constants defined above and

\footnotetext{
${ }^{16}$ The weighted sum $y$ is also normally distributed with mean $\mu_{y}$ and variance $\sigma_{y}^{2}$, which corresponds to $\Sigma_{22}$ as calculated below.

${ }^{17}$ See Greene (2003), Appendix B.11.
} 
$\Sigma$ is the covariance matrix of $x$ and $z$, i.e.,

$$
\Sigma=\left(\begin{array}{cc}
\sigma_{x}^{2} & \sigma_{x} \sigma_{z} \\
\sigma_{x} \sigma_{z} & \sigma_{z}^{2}
\end{array}\right)
$$

So the covariance matrix of vector $(x, z, y)^{\prime}$ is given by

$$
\hat{\Sigma}=\left(\begin{array}{ccc}
\sigma_{x}^{2} & \sigma_{x} \sigma_{z} & a \sigma_{x}^{2}+b \sigma_{x} \sigma_{z} \\
\sigma_{x} \sigma_{z} & \sigma_{z}^{2} & a \sigma_{x} \sigma_{z}+b \sigma_{z}^{2} \\
a \sigma_{x}^{2}+b \sigma_{x} \sigma_{z} & a \sigma_{x} \sigma_{z}+b \sigma_{z}^{2} & a^{2} \sigma_{x}^{2}+b^{2} \sigma_{z}^{2}+2 a b \sigma_{x} \sigma_{z}
\end{array}\right) .
$$

So, we have a set of normally distributed variables $(x, z, y)$ with mean vector $\left(\mu_{x}, \mu_{z}, \mu_{y}\right)$ and covariance matrix $\hat{\Sigma}$, which we will partition into two subsets, namely in vector $(x, z)^{\prime}$ and $(y)$. The mean vector and the covariance matrix $\hat{\Sigma}$ are also partitioned accordingly, such that we can rewrite matrix $\hat{\Sigma}$ as

$$
\hat{\Sigma}=\left(\begin{array}{ll}
\Sigma_{11} & \Sigma_{12} \\
\Sigma_{21} & \Sigma_{22}
\end{array}\right)
$$

where

$$
\Sigma_{12}:=\left(\begin{array}{c}
a \sigma_{x}^{2}+b \sigma_{x} \sigma_{z} \\
a \sigma_{x} \sigma_{z}+b \sigma_{z}^{2}
\end{array}\right)
$$

and

$$
\Sigma_{22}:=\left(a^{2} \sigma_{x}^{2}+b^{2} \sigma_{z}^{2}+2 a b \sigma_{x} \sigma_{z}\right) .
$$

Following the above-mentioned theorem, the expectation of $x$, respectively $z$, given the observed value of $y$ then is

$$
\begin{aligned}
E\left[\left(\begin{array}{l}
x \\
z
\end{array}\right) \mid y\right] & \left.=\left(\begin{array}{l}
\mu_{x} \\
\mu_{z}
\end{array}\right)+\Sigma_{12} \Sigma_{22}^{-1}\left(y-\mu_{y}\right)\right) \\
& =\left(\begin{array}{l}
\mu_{x} \\
\mu_{z}
\end{array}\right)+\left(\begin{array}{c}
a \sigma_{x}^{2}+b \sigma_{x} \sigma_{z} \\
a \sigma_{x} \sigma_{z}+b \sigma_{z}^{2}
\end{array}\right) \cdot \frac{y-a \mu_{x}-b \mu_{z}}{a^{2} \sigma_{x}^{2}+b^{2} \sigma_{z}^{2}+2 a b \sigma_{x} \sigma_{z}}
\end{aligned}
$$

This can, in turn, be used to calculate the worker's expected output in the second job, given his performance in the first.

\section{With Independently Distributed Skills}

If $x, z$ are independently distributed, their covariance is zero, and the conditional expectation of $x$ and $z$ given $y$ then simplifies to

$$
E\left[\left(\begin{array}{c}
x \\
z
\end{array}\right) \mid y\right]=\left(\begin{array}{c}
\mu_{x} \\
\mu_{z}
\end{array}\right)+\left(\begin{array}{c}
a \sigma_{x}^{2} \\
b \sigma_{z}^{2}
\end{array}\right)\left(\frac{1}{a^{2} \sigma_{x}^{2}+b^{2} \sigma_{z}^{2}}\right)\left(y-a \mu_{x}-b \mu_{z}\right)
$$




\section{References}

Agrawal, A., C. R. Knoeber, and T. Tsoulouhas (2006): "Are outsiders handicapped in CEO successions?," Journal of Corporate Finance, 12(3), 619 - 644, Corporate Governance.

Antonovics, K., and L. Golan (2007): "Experimentation and Job Choice," mimeo.

Campion, M., L. Cheraskin, and M. Stevens (1994): "Career-Related Antecedents and Outcomes of Job Rotation," The Academy of Management Journal, 37(6), 1518-1542.

Chan, W. (1996): "External Recruitment versus Internal Promotion," Journal of Labor Economics, 14(4), 555-570.

Demougin, D., and A. Siow (1994): "Careers in Ongoing Hierarchies," American Economic Review, 84(5), 1261-1277.

DeVaro, J., and H. Morita (2009): "Internal Promotion and External Recruitment: A Theoretical and Empirical Analysis," mimeo.

Eriksson, T., and J. Ortega (2006): "The Adoption of Job Rotation: Testing the Theories," Industrial and Labor Relations Review, 59(4), 653666.

Fairburn, J., and J. Malcomson (2001): "Performance, Promotion, and the Peter Principle," Review of Economic Studies, 68(1), 45-66.

Faria, J. (2000): "An Economic Analysis of the Peter and Dilbert Principles," Working Paper No. 101 University of Technology Sydney.

Gathmann, C., and U. Schonberg (2010): "How General Is Human Capital? A Task-Based Approach," Journal of Labor Economics, 28(1), $1-49$.

Gibbons, R., and M. Waldman (2004): "Task-Specific Human Capital," American Economic Review, 94(2), 203-207.

- (2006): "Enriching a Theory of Wage and Promotion Dynamics inside Firms," Journal of Labor Economics, 24(1), 59-107.

Greene, W. (2003): Econometric Analysis. Pearson Education, New Jersey, US, 5th international edn. 
Greenwald, B. C. (1986): "Adverse Selection in the Labour Market," The Review of Economic Studies, 53(3), 325-347.

Grossman, S., R. Kinlstrom, and L. Mirman (1977): "A Bayesian Approach to the Production of Information and Learning by Doing," Review of Economic Studies, 44(3), 533-547.

Hutchens, R. (2006): "Job Opportunities for Older Workers: When Are Jobs Filled With External Hires?," Discussion paper, ILR School Cornell University.

Kusunoki, K., And T. Numagami (1998): "Interfunctional Transfers of Engineers in Japan: Empirical Findings and Implications for Cross Sectional Integration," IEEE Transactions on Engineering Management, 45(3), 250-262.

Lauterbach, B., J. Vu, And J. Weisberg (1999): "Internal vs. External Successions and Their Effect on Firm Performance," Human Relations, 52(12), 1485-1504.

LazeAr, E. (2004): "The Peter Principle: A Theory of Decline," Journal of Political Economy, 112(1), 141-163.

OrtegA, J. (2001): "Job Rotation as a Learning Mechanism," Management Science, 47(10), 1361-1370.

Peter, L., And R. Hull (1969): The Peter Principle: Why Things Always Go Wrong. Morrow, New York.

Sattinger, M. (1993): "Assignment Models of the Distribution of Earnings," Journal of Economic Literature, 31(2), 831-880.

VAlsecChi, I. (2000): "Job Assignment and Promotion," Journal of Economic Surveys, 14(1), 31-51.

Waldman, M. (2003): "Ex ante versus ex post optimal promotion rules: The case of internal promotion," Economic Inquiry, 41(1), 27-41. 